\title{
GLUING FORMULAE FOR DONALDSON INVARIANTS FOR CONNECTED SUMS ALONG SURFACES*
}

\author{
VICENTE MUÑOZ ${ }^{\dagger}$
}

\begin{abstract}
Following our work in [18], we prove a gluing formula for the Donaldson invariants of the connected sum of two four-manifolds along surfaces of the same genus $g$, self-intersection zero and representing odd homology classes, solving a conjecture of Morgan and Szabó [14].
\end{abstract}

1. Introduction. This paper tries to answer the question of the behaviour of the Donaldson invariants under connected sums along surfaces of arbitrary genus $g \geq 2$ and self-intersection zero. The problem was first motivated by the computation of the basic classes of elliptic surfaces. The general elliptic surface with $b^{+}>1$ and simply connected, is constructed by the process of connected sum along embedded tori of self-intersection zero from elementary pieces (rational elliptic surfaces and homotopy $K 3$-surfaces). After establishing the appropriate gluing formula for the behaviour of Donaldson invariants under the operation of connected sum along embedded tori, the information of the basic classes for some particular examples gives the basic classes of any such elliptic surface. This has been carried out by many authors [13] [3] [17] [15] [19].

The following natural case is the connected sum along embedded surfaces of genus $g=2$. Morgan and Szabó [20] [14] treated the case when the self-intersection of one of the surfaces is 1 (and the other -1). The author [18] [17] has solved the case in which the surfaces have self-intersection zero and are odd in homology, giving a number of nice applications. For this he used the Fukaya-Floer homology as developed in [1].

The case we are going to deal with in this paper is the connected sum along embedded surfaces of higher (arbitrary) genus $g$, self-intersection zero and odd, giving a positive answer to the conjectures in the literature $[14$, conjecture 7.2$][17$, conjecture $5.27]$.

Let $X$ be a smooth, compact, oriented four-manifold with $b^{+}>1$ and $b^{+}-b_{1}$ odd. For any $w \in H^{2}(X ; \mathbb{Z}), D_{X}^{w}$ will denote the corresponding Donaldson invariant [4] [12], which is defined as a linear functional on $\mathbb{A}(X)=\operatorname{Sym}^{*}\left(H_{0}(X) \oplus H_{2}(X)\right) \otimes \bigwedge^{*} H_{1}(X)$ $\left(H_{*}(X)\right.$ will always denote homology with rational coefficients, and similarly for $\left.H^{*}(X)\right)$. Let $x \in H_{0}(X)$ be the class of a point. Then Kronheimer and Mrowka [12] define $X$ to be of simple type (with respect to $w$ ) when $D_{X}^{w}\left(\left(x^{2}-4\right) z\right)=0$ for all $z \in \mathbb{A}(X)$, and in that case define

$$
\mathbb{D}_{X}^{w}(z)=D_{X}^{w}\left(\left(1+\frac{x}{2}\right) z\right)
$$

for all $z \in \operatorname{Sym}^{*} H_{2}(X)$. The series $\mathbb{D}_{X}^{w}\left(e^{t \alpha}\right), \alpha \in H_{2}(X)$, is even or odd depending on whether

$$
d_{0}=d_{0}(X, w)=-w^{2}-\frac{3}{2}\left(1-b_{1}+b^{+}\right)
$$

is even or odd. When $b_{1}=0$ and $b^{+}>1, X$ is of simple type with respect to some $w$ if and only if it is so with respect to any $w$. In such case, $X$ is just called of simple type.

*Received February 4, 1998; accepted for publication February 19, 1998.

†Departamento de Álbegra, Geometría y Topología, Facultad de Ciencias, Universidad de Málaga, AP. 59, 29080 Málaga, Spain (vmunoz@agt.cie.uma.es). 
Proposition 1 ([12]). Let $X$ be a manifold of simple type with $b_{1}=0$ and $b^{+}>1$ and odd. Then we have

$$
\mathbb{D}_{X}^{w}\left(e^{\alpha}\right)=e^{Q(\alpha) / 2} \sum a_{j, w} e^{K_{j} \cdot \alpha}
$$

for finitely many $K_{j} \in H^{2}(X ; \mathbb{Z})$ (called basic classes) and $a_{j, w}=(-1)^{\frac{K_{j} \cdot w+w^{2}}{2}} a_{j}$, where $a_{j}$ are rational numbers (the collection is empty when the invariants all vanish). These classes are lifts to integral cohomology of $w_{2}(X)$. Moreover, for any embedded surface $\Sigma \hookrightarrow X$ of genus $g$ and with $\Sigma^{2} \geq 0$, one has $2 g-2 \geq \Sigma^{2}+\left|K_{j} \cdot \Sigma\right|$.

DEFINITION 2 ([18]). $(w, \Sigma)$ is an allowable pair if $w, \Sigma \in H^{2}(X ; \mathbb{Z}), w \cdot \Sigma \equiv 1$ $(\bmod 2)$ and $\Sigma^{2}=0$. Then we define

$$
D_{X}^{(w, \Sigma)}=D_{X}^{w}+D_{X}^{w+\Sigma}
$$

When $b^{+}=1$, the invariants depend on the metric through a structure of walls and chambers [10] and therefore we have to specify the metric. As $w \cdot \Sigma \equiv 1(\bmod 2)$, we have that $\Sigma$ is contained in the closure of a unique chamber of type $\left(w, p_{1}\right)$ for every $p_{1}<0, p_{1} \equiv w^{2} \quad(\bmod 4)$. So, in the case that the invariants only depend on the metric through the period point (for instance, simply-connected manifolds [11] and $\Sigma \times \mathbb{C P}^{1}$, with $\Sigma$ a Riemann surface, which are all the cases we need for our arguments), we shall consider the invariants referring to the chambers defined by $\Sigma$.

The series $D_{X}^{(w, \Sigma)}\left(e^{t \alpha}\right), \alpha \in H_{2}(X)$, is even or odd according to whether $d_{0}$ is even or odd. Since $(w+\Sigma)^{2} \equiv w^{2}+2 \quad(\bmod 4)$, we can recover $D_{X}^{w}$ and $D_{X}^{w+\Sigma}$ from $D_{X}^{(w, \Sigma)}$.

Proposition 3 ([18]). Suppose $X$ is a manifold of simple type with $b_{1}=0$ and $b^{+}>1$ and odd. Write the Donaldson series as $\mathbb{D}_{X}^{w}\left(e^{\alpha}\right)=e^{Q(\alpha) / 2} \sum a_{j, w} e^{K_{j} \cdot \alpha}$. Then setting $d_{0}=d_{0}(X, w)=-w^{2}-\frac{3}{2}\left(1+b^{+}\right)$we have

$$
D_{X}^{(w, \Sigma)}\left(e^{\alpha}\right)=e^{Q(\alpha) / 2} \sum_{K_{j} \cdot \Sigma \equiv 2} a_{j, w} e^{K_{j} \cdot \alpha}+e^{-Q(\alpha) / 2} \sum_{K_{j} \cdot \Sigma \equiv 0} i_{(\bmod 4)}^{-d_{0}} a_{j, w} e^{i K_{j} \cdot \alpha}
$$

So giving $\mathbb{D}_{X}^{w}$ is equivalent to giving $D_{X}^{(w, \Sigma)}$.

REMARK 4. Under the conditions of proposition 3, we can prove in a similar fashion that

$$
D_{X}^{(w, \Sigma)}\left(x e^{\alpha}\right)=2 e^{Q(\alpha) / 2} \sum_{K_{j} \cdot \Sigma \equiv 2} a_{j, w} e^{K_{j} \cdot \alpha}-2 e^{-Q(\alpha) / 2} \sum_{K_{j} \cdot \Sigma \equiv 0} i_{(\bmod 4)}^{-d_{0}} a_{j, w} e^{i K_{j} \cdot \alpha}
$$

and using that $D_{X}^{(w, \Sigma)}\left(x^{a} e^{\alpha+\Sigma}\right)=\sum \frac{1}{b !} D_{X}^{(w, \Sigma)}\left(\Sigma^{b} x^{a} e^{\alpha}\right)$, one has

$$
\begin{aligned}
D_{X}^{(w, \Sigma)}\left(\Sigma^{b} x^{a} e^{\alpha}\right)= & e^{Q(\alpha) / 2} \sum_{K_{j} \cdot \Sigma \equiv 2} a_{j, w} 2^{a}\left(\left(\alpha+K_{j}\right) \cdot \Sigma\right)^{b} e^{K_{j} \cdot \alpha}+ \\
& +e^{-Q(\alpha) / 2} \sum_{K_{j} \cdot \Sigma \equiv 0} i^{-d_{0}} a_{j, w}(-2)^{a}\left(\left(-\alpha+i K_{j}\right) \cdot \Sigma\right)^{b} e^{i K_{j} \cdot \alpha}
\end{aligned}
$$

Definition 5 (compare [18]). We say that $(X, \Sigma)$ is permissible if $X$ is a smooth compact oriented four-manifold and $\Sigma \hookrightarrow X$ is an embedded Riemann surface of genus $g \geq 2$ and self-intersection zero such that $[\Sigma] \in H_{2}(X ; \mathbb{Z})$ is odd (its reduction modulo 2 is non-zero, or equivalently, it is an odd multiple of a primitive homology 
class). So we can consider $w \in H^{2}(X ; \mathbb{Z})$ with $w \cdot \Sigma \equiv 1 \quad(\bmod 2)$. Then $(w, \Sigma)$ is an allowable pair (we consider $\Sigma \in H^{2}(X ; \mathbb{Z})$ through Poincaré duality). This implies that $b^{+}>0$. Let $N_{\Sigma} \cong A=\Sigma \times D^{2}$ be a tubular neighbourhood of $\Sigma$ and set $X^{o}=X-\operatorname{int}\left(N_{\Sigma}\right)$. Then $\partial X^{o}=Y \cong \Sigma \times \mathbb{S}^{1}$ (but the isomorphism is not canonical). We consider one such isomorphism fixed and (when necessary) we furnish $X^{\circ}$ with $a$ cylindrical end, i.e. we consider $X_{c e}^{o}=X^{o} \cup(Y \times[0, \infty))$.

We call identification for $Y=\Sigma \times \mathbb{S}^{1}$ any (orientation preserving) bundle automorphism $\phi: Y \stackrel{\sim}{\rightarrow} Y$. Up to isotopy, $\phi$ depends only on the isotopy class of the induced diffeomorphism on $\Sigma$ and on an element of $H^{1}(\Sigma ; \mathbb{Z})$.

Definition 6 ([18]). Let $\left(X_{1}, \Sigma_{1}\right)$ and $\left(X_{2}, \Sigma_{2}\right)$ be permissible, with $\Sigma_{1}$ and $\Sigma_{2}$ of the same genus $g$. We pick orientations so that $\partial X_{1}^{o}=-\partial X_{2}^{o}=Y$ (minus means reversed orientation). Then $X=X(\phi)=X_{1}^{o} \cup_{\phi} X_{2}^{o}=X_{1} \#_{\Sigma} X_{2}$ is called the connected sum along $\Sigma$ of $\left(X_{1}, \Sigma_{1}\right)$ and $\left(X_{2}, \Sigma_{2}\right)$ (with identification $\phi$ ). It is a compact, naturally oriented, smooth four-manifold with an embedding $Y \hookrightarrow X$ such that $X-Y=\left(X_{1}^{o}\right)_{c e} \sqcup\left(X_{2}^{o}\right)_{c e} \subset X_{1} \sqcup X_{2}$.

The induced homology classes $\left[\Sigma_{1}\right]$ and $\left[\Sigma_{2}\right]$ coincide and are induced by an embedded $\Sigma \hookrightarrow X$. Then $(X, \Sigma)$ is permissible. Choose $w_{i} \in H^{2}\left(X_{i} ; \mathbb{Z}\right), i=1,2$, and $w \in H^{2}(X ; \mathbb{Z}) \operatorname{such}$ that $w_{i} \cdot \Sigma_{i} \equiv 1 \quad(\bmod 2), w \cdot \Sigma \equiv 1 \quad(\bmod 2)$, in a compatible way (i.e. the restricition of $w$ to $X_{i}^{o} \subset X$ coincides with the restriction of $w_{i}$ to $X_{i}^{o} \subset X_{i}$ ). We have $w^{2} \equiv w_{1}^{2}+w_{2}^{2} \quad(\bmod 2)$. Changing $w$ by $w+\Sigma$ if necessary, we can always suppose $w^{2} \equiv w_{1}^{2}+w_{2}^{2} \quad(\bmod 4)$. In general, we shall call $w$ all of them, not making explicit to which manifold they refer. Note also that if $b_{1}\left(X_{1}\right)=b_{1}\left(X_{2}\right)=0$ then $b_{1}(X)=0$ and $b^{+}(X)>1$.

REMARK 7. There is a case when there is a preferred identification. Suppose $X_{1}$ and $X_{2}$ are complex surfaces, $\Sigma$ is a complex curve of genus $g$ and there are holomorphic embeddings $\Sigma \hookrightarrow X_{i}$, with image $\Sigma_{i}, i=1,2$, such that $\left[\Sigma_{i}\right]$ is odd and has self-intersection zero. Then the holomorphic normal bundle to $\Sigma_{i}$ gives a preferred isomorphism $\partial N_{\Sigma_{i}} \stackrel{\sim}{\rightarrow} \Sigma_{i} \times \mathbb{S}^{1} \cong \Sigma \times \mathbb{S}^{1}$, and hence a preferred identification $\partial X_{1}^{o} \stackrel{\sim}{\rightarrow}-\partial X_{2}^{o}$.

REMARK 8. Let $X_{1}$ and $X_{2}$ be as in definition 6 and $X=X_{1} \#_{\Sigma} X_{2}$. Then homology orientations for $X_{1}$ and $X_{2}$ induce canonically a homology orientation for $X$. To see this, define $H_{+}^{2}\left(X_{i}^{o}\right)$ to be a maximal definite positive subspace for the intersection pairing of $X_{i}^{o}$ restricted to the image of $H_{2}\left(X_{i}^{o}\right) \rightarrow H_{2}\left(X_{i}^{o}, \partial X_{i}^{o}\right) \cong$ $H^{2}\left(X_{i}^{o}\right)$. Then it is easy to see that the exact sequence for the pair $\left(X_{i}, X_{i}^{o}\right)$ and the Mayer-Vietoris sequence for $X=X_{1}^{o} \cup X_{2}^{o}$ yield the following exact sequences

$$
\begin{gathered}
0 \rightarrow H^{1}\left(X_{i}\right) \rightarrow H^{1}\left(X_{i}^{o}\right) \rightarrow H^{2}(A, \partial A) \rightarrow H_{+}^{2}\left(X_{i}\right) \rightarrow H_{+}^{2}\left(X_{i}^{o}\right) \rightarrow 0 \\
0 \rightarrow H^{1}(X) \rightarrow H^{1}\left(X_{1}^{o}\right) \oplus H^{1}\left(X_{2}^{o}\right) \rightarrow H^{1}(Y) \rightarrow H_{+}^{2}(X) \rightarrow H_{+}^{2}\left(X_{1}^{o}\right) \oplus H_{+}^{2}\left(X_{2}^{o}\right) \rightarrow 0,
\end{gathered}
$$

where $A=\Sigma \times D^{2}$ as before. We fix an orientation of $H^{1}(Y)$ (it does not change when we reverse the orientation of $Y$ ). So homology orientations for $X_{1}$ and $X_{2}$ induce a homology orientation for $X$.

Let $\mathcal{H}=\left\{D \in H_{2}(X) /\left.D\right|_{Y}=k\left[\mathbb{S}^{1}\right] \in H_{1}(Y)\right.$, some $\left.k \in \mathbb{Q}\right\}$. This subspace of $H_{2}(X)$ contains the image of $H_{2}\left(X_{1}^{o}\right) \oplus H_{2}\left(X_{2}^{o}\right)$. For every $D \in \mathcal{H}$, choose $D_{i} \in H_{2}\left(X_{i}\right)$ agreeing with $D$ (i.e. $\left.\left.D_{i}\right|_{X_{i}^{\circ}}=\left.D\right|_{X_{i}^{\circ}}, i=1,2\right)$ and with $D^{2}=D_{1}^{2}+D_{2}^{2}$. Moreover, we can suppose that the map $D \mapsto\left(D_{1}, D_{2}\right)$ is linear. Actually, once chosen one of such maps, any other is of the form $D \mapsto\left(D_{1}+r \Sigma, D_{2}-r \Sigma\right)$, for a (rational) number $r$. Let us state now our main result. 
Theorem 9. Suppose $\left(X_{1}, \Sigma_{1}\right)$ and $\left(X_{2}, \Sigma_{2}\right)$ are permissible with $\Sigma_{1}$ and $\Sigma_{2}$ of the same genus $g \geq 2$. Suppose also that $X_{1}, X_{2}$ have both $b_{1}=0$ and $b^{+}>1$ and are of simple type. Let

$$
\mathbb{D}_{X_{1}}^{w}\left(e^{\alpha}\right)=e^{Q(\alpha) / 2} \sum a_{j, w} e^{K_{j} \cdot \alpha}
$$

and

$$
\mathbb{D}_{X_{2}}^{w}\left(e^{\alpha}\right)=e^{Q(\alpha) / 2} \sum b_{k, w} e^{L_{k} \cdot \alpha}
$$

Choose any identification $\phi$ and let $X=X(\phi)=X_{1} \#_{\Sigma} X_{2}$ be the connected sum along $\Sigma$, with the induded homology orientation. Suppose finally that $X$ is of simple type. Let $w \in H^{2}(X ; \mathbb{Z}), w_{i} \in H^{2}\left(X_{i} ; \mathbb{Z}\right), i=1,2$, in a compatible way, such that $w_{i} \cdot \Sigma_{i} \equiv 1$ $(\bmod 2), w \cdot \Sigma \equiv 1 \quad(\bmod 2)$ and $w^{2} \equiv w_{1}^{2}+w_{2}^{2} \quad(\bmod 4)$. For every $D \in \mathcal{H}$, we choose $D_{i} \in H_{2}\left(X_{i}\right)$ agreeing with $D$ and satisfying $D^{2}=D_{1}^{2}+D_{2}^{2}$ in such a way that the map $D \mapsto\left(D_{1}, D_{2}\right)$ is linear. Then

$$
\begin{gathered}
\mathbb{D}_{X}^{w}\left(e^{t D}\right)=e^{Q(t D) / 2}\left(\sum_{K_{j} \cdot \Sigma=L_{k} \cdot \Sigma=2 g-2}-2^{7 g-9} a_{j, w} b_{k, w} e^{\left(K_{j} \cdot D_{1}+L_{k} \cdot D_{2}+2 \Sigma \cdot D\right) t}+\right. \\
\left.+\sum_{K_{j} \cdot \Sigma=L_{k} \cdot \Sigma=-(2 g-2)}(-1)^{g} 2^{7 g-9} a_{j, w} b_{k, w} e^{\left(K_{j} \cdot D_{1}+L_{k} \cdot D_{2}-2 \Sigma \cdot D\right) t}\right)
\end{gathered}
$$

REMARK 10. If we do not assume $w^{2} \equiv w_{1}^{2}+w_{2}^{2} \quad(\bmod 4)$, we get an extra factor $\epsilon=(-1)^{(g-1)\left(w^{2}-w_{1}^{2}-w_{2}^{2}\right) / 2}$ in front of formula (1).

REMARK 11. The reason for the sign is easy to work out. First, $w^{2} \equiv w_{1}^{2}+w_{2}^{2}$ $(\bmod 2)$. Also,

$$
-\frac{3}{2}\left(1-b_{1}(X)+b^{+}(X)\right)=-\frac{3}{2}\left(1-b_{1}\left(X_{1}\right)+b^{+}\left(X_{1}\right)\right)-\frac{3}{2}\left(1-b_{1}\left(X_{2}\right)+b^{+}\left(X_{2}\right)\right)-3(g-1) .
$$

Therefore, $d_{0}(X, w) \equiv d_{0}\left(X_{1}, w\right)+d_{0}\left(X_{2}, w\right)+g-1 \quad(\bmod 2)$. Now the sign comes from the fact that the coefficient for the basic class $-\kappa$ is $(-1)^{d_{0}} c_{\kappa}$, being $c_{\kappa}$ the coefficient for the basic class $\kappa$.

REMARK 12. If we are in the conditions of theorem 9 , but $g=1$, we get a slightly different answer [17, chapter 4] [14]. For all basic classes, it is $K_{j} \cdot \Sigma=L_{k} \cdot \Sigma=0$, and

$$
\begin{gathered}
\mathbb{D}_{X}^{w}\left(e^{t D}\right)=e^{Q(t D) / 2}\left(\sum_{K_{j}, L_{k}}-\frac{1}{4} a_{j, w} b_{k, w} e^{\left(K_{j} \cdot D_{1}+L_{k} \cdot D_{2}+2 \Sigma \cdot D\right) t}+\right. \\
\left.+\sum_{K_{j}, L_{k}}-\frac{1}{4} a_{j, w} b_{k, w} e^{\left(K_{j} \cdot D_{1}+L_{k} \cdot D_{2}-2 \Sigma \cdot D\right) t}+\sum_{K_{j}, L_{k}}-\frac{1}{2} a_{j, w} b_{k, w} e^{\left(K_{j} \cdot D_{1}+L_{k} \cdot D_{2}\right) t}\right)
\end{gathered}
$$

Corollary 13. Suppose we are in the conditions of theorem 9. Write $\mathbb{D}_{X}\left(e^{\alpha}\right)=$ $e^{Q(\alpha) / 2} \sum c_{\kappa} e^{\kappa \cdot \alpha}, \mathbb{D}_{X_{1}}\left(e^{\alpha}\right)=e^{Q(\alpha) / 2} \sum a_{j} e^{K_{j} \cdot \alpha}$ and $\mathbb{D}_{X_{2}}\left(e^{\alpha}\right)=e^{Q(\alpha) / 2} \sum b_{k} e^{L_{k} \cdot \alpha}$ for the Donaldson series for $X, X_{1}$ and $X_{2}$, respectively. Then given any pair $(K, L) \in$ $H^{2}\left(X_{1}^{o} ; \mathbb{Z}\right) \oplus H^{2}\left(X_{2}^{o} ; \mathbb{Z}\right)$, we have

$$
\sum_{\left\{\kappa /\left.\kappa\right|_{X_{1}^{o}}=K,\left.\kappa\right|_{X_{2}^{o}}=L\right\}} c_{\kappa}=( \pm 1)^{g-1} 2^{7 g-9}\left(\sum_{\left.K_{j}\right|_{X_{1}^{o}=K}} a_{j}\right) \cdot\left(\sum_{\left.L_{k}\right|_{X_{2}^{o}}=L} b_{k}\right)
$$


whenever $\left.K\right|_{Y}=\left.L\right|_{Y}= \pm(2 g-2)$ P.D. $\left[\mathbb{S}^{1}\right]$. Otherwise, the left hand side is zero.

Proof. Allowing $D$ to vary in $\mathcal{H}$, formula (1) gives

$$
\sum_{\left\{\kappa /\left.\kappa\right|_{X_{1}^{\circ}}=K,\left.\kappa\right|_{X_{2}^{\circ}}=L\right\}} c_{\kappa, w} e^{(\kappa \cdot D) t}=\sum_{\substack{\left.\left.\kappa_{j}\right|_{X_{1}^{\circ}=K} \\ L_{k}\right|_{X_{2}^{\circ}}=L}}-( \pm 1)^{g-1} 2^{7 g-9} a_{j, w} b_{k, w} e^{\left(K_{j} \cdot D_{1}+L_{k} \cdot D_{2} \pm 2 \Sigma \cdot D\right) t} .
$$

(2)

We cannot have more precise information on $\kappa$ as we cannot evaluate $\mathbb{D}_{X}^{w}$ on all $D \in H_{2}(X)$. Now take $w=$ P.D. $[D] \in H^{2}(X ; \mathbb{Z}), w_{1}=$ P.D. $\left[D_{1}\right] \in H^{2}\left(X_{1} ; \mathbb{Z}\right)$ and $w_{2}=$ P.D. $\left[D_{2}\right] \in H^{2}\left(X_{2} ; \mathbb{Z}\right)$ with $w^{2}=w_{1}^{2}+w_{2}^{2}$ and $w \cdot \Sigma=1$. Substitute $t=\pi i / 2$ in (2) and multiply by $(-1)^{w^{2} / 2}=(-1)^{w_{1}^{2} / 2}(-1)^{w_{2}^{2} / 2}$, to get the sought expression.

REMARK 14. In theorem 9 we cannot hope for having a similar formula for classes $D$ such that $\left.D\right|_{Y}$ is not a multiple of $\left[\mathbb{S}^{1}\right]$ in $H_{1}(Y)$. This is due to the fact that we cannot find $D_{i} \in H_{2}\left(X_{i}\right)$ agreeing with $D$ (i.e. $\left.D_{i}\right|_{X_{i}^{\circ}}=\left.D\right|_{X_{i}^{\circ}}$, for $i=1,2$ ). We would need to relate the invariant $\mathbb{D}_{X}^{w}\left(e^{t D}\right)$ with invariants of the form $\mathbb{D}_{\tilde{X}_{i}}^{w}\left(e^{t D_{i}}\right)$, for suitable manifolds $\tilde{X}_{i}$ containing $X_{i}^{o}, D_{i} \in H_{2}\left(\tilde{X}_{i}\right)$, such that $\left.D_{i}\right|_{X_{i}^{\circ}}=\left.D\right|_{X_{i}^{\circ}}$. This was done in [18, theorem 10] for the case $g=2$ (see also conjecture in section 5).

This limitation prevents us from having more general results. For example, we do not know whether (under the conditions of theorem 9) there are basic classes $\kappa$ for $X=X_{1} \#_{\Sigma} X_{2}$ such that $|\kappa \cdot \Sigma|<2 g-2$ or not (compare [18, corollary 11]).

In some cases, theorem 9 is all that we need to find explicitly the basic classes for $X$. This is due to the fact that there is a subspace $V \subset H_{2}(X)$ where all the basic classes vanish, such that $H_{2}(X)=\mathcal{H} \oplus V$. In [14, definition 4.1], Morgan and Szabó define admissible identification, which is a condition which implies that $X=X_{1} \#_{\Sigma} X_{2}$ is of simple type and such $V$ exists.

CoROllary 15. Suppose there exists a subspace $V \subset H_{2}(X)$ where all the basic classes vanish such that $H_{2}(X)=\mathcal{H} \oplus V$. Then there are no basic classes $\kappa$ for $X$ such that $|\kappa \cdot \Sigma|<2 g-2$. The basic classes for $X$ are indexed by pairs of basic classes $\left(K_{i}, L_{j}\right)$ for $X_{1}$ and $X_{2}$ respectively, such that $K_{i} \cdot \Sigma=L_{j} \cdot \Sigma= \pm(2 g-2)$.

REMARK 16. Corollary 13 agrees with the results of the kind for the SeibergWitten invariants [17, section 7.3]. Morgan, Szabó and Taubes [16] have proved the analogous result to corollary 13 for the Seiberg-Witten basic classes (not the part corresponding to basic classes $\kappa$ for $X$ with $|\kappa \cdot \Sigma|<2 g-2$ ). Both results are equivalent supposing true the conjecture of Witten [22] about the relationship of Donaldson and Seiberg-Witten invariants.

Our last result is

Theorem 17. Let $S=\Sigma \times \mathbb{C P}^{1}, w=$ P.D. $\left[\mathbb{C P}^{1}\right] \in H^{2}(S ; \mathbb{Z})$. Suppose that $S$ is of finite type of order $n \geq 1$ with respect to $w$ and $w+\Sigma$ for the metrics defined by $\Sigma$ (i.e. $D_{S}^{(w, \Sigma)}\left(\left(x^{2}-4\right)^{n} z\right)=0$, for all $\left.z \in \mathbb{A}(S)\right)$. Then for any $(X, \Sigma)$ permissible, $X$ is of finite type of order at most $n$ with respect to any $w \in H^{2}(X ; \mathbb{Z})$ with $w \cdot \Sigma \equiv 1$ ( $\bmod 2)$ (and for metrics defined by $\Sigma$, in the case $b^{+}=1$ ).

2. Gluing theory. Now we are going to develop the gluing theory necessary to prove theorem 9. The set up is as follows, $X=X_{1}^{o} \cup_{Y} X_{2}^{o}$, where $\partial X_{1}^{o}=-\partial X_{2}^{o}=Y$, an oriented three-manifold (later $\left.Y=\Sigma \times \mathbb{S}^{1}\right), w \in H^{2}(X ; \mathbb{Z}), w_{i}=\left.w\right|_{X_{i}} \in H^{2}\left(X_{i}^{o} ; \mathbb{Z}\right)$, and $D \in H_{2}(X)$ a homology class. So $\left.D\right|_{Y} \in H_{1}(Y)$. We want to evaluate $D_{X}^{w}\left(D^{d}\right)$, the invariant being linear, we may multiply $D$ by any non-zero rational number, and hence suppose that $\left.D\right|_{Y}$ is either zero or primitive in $H_{1}(Y ; \mathbb{Z})$. Now we represent $D$ by a cycle $D \subset X$ and put $D_{i}^{o}=D \cap X_{i}^{o}$, which we shall write formally as $D=D_{1}^{o}+D_{2}^{o}$, $D_{i}^{o} \subset X_{i}^{o}$. We can suppose $\partial D_{1}^{o}=-\partial D_{2}^{o}=\gamma$, with $\gamma \subset Y$ an embedded curve in $Y$, 
so when we give $X_{1}^{o}$ a cylindrical end, we have $D_{1}^{o} \cap(Y \times[0, \infty))=\gamma \times[0, \infty)$ (and analogously for $X_{2}^{o}$ ).

Proposition 18 ([2] [1] [17]). Suppose $\left.w\right|_{Y}$ odd. Then we have one of the following cases:

- $\left.D\right|_{Y}=0$ in $H_{1}(Y ; \mathbb{Z})$. Represent $D$ by a cycle so $D=D_{1}^{o}+D_{2}^{o}, D_{i}^{o} \subset$ $X_{i}^{o}, \partial D_{1}^{o}=\partial D_{2}^{o}=\emptyset$. Consider the Floer homology groups [2] $H F_{*}(Y)$ (graded mod 4). Then $\left(X_{i}^{o}, D_{i}^{o}\right)$ define relative invariants $\phi^{w_{1}}\left(X_{1}^{o}, e^{t D_{1}^{o}}\right) \in$ $H F_{*}(Y) \otimes \mathbb{Q}[[t]], \phi^{w_{2}}\left(X_{2}^{o}, e^{t D_{2}^{o}}\right) \in H F_{*}(-Y) \otimes \mathbb{Q}[[t]]$. There is a natural pairing $H F_{*}(Y) \otimes H F_{*}(-Y) \rightarrow \mathbb{Q}$, such that

$$
D_{X}^{(w, \Sigma)}\left(e^{t D}\right)=<\phi^{w_{1}}\left(X_{1}^{o}, e^{t D_{1}^{o}}\right), \phi^{w_{2}}\left(X_{2}^{o}, e^{t D_{2}^{o}}\right)>.
$$

- $\left.D\right|_{Y} \neq 0$. Substitute $D$ by a rational multiple if necessary so that $\left.D\right|_{Y} \in$ $H_{1}(Y ; \mathbb{Z})$ is a primitive element in $H_{1}(Y ; \mathbb{Z})$. Represent $D$ by a cycle so $D=D_{1}^{o}+D_{2}^{o}, D_{i}^{o} \subset X_{i}^{o}, \partial D_{1}^{o}=-\partial D_{2}^{o}=\gamma$, with $\gamma \subset Y$ an embedded curve in

$Y$. Consider the Fukaya-Floer homology groups [1] $\mathrm{HFF}_{*}(Y, \gamma)$ (graded mod 4). Then $\left(X_{i}^{o}, D_{i}^{o}\right)$ define relative invariants $\phi^{w_{1}}\left(X_{1}^{o}, e^{t D_{1}^{o}}\right) \in H F F_{*}(Y, \gamma)$, $\phi^{w_{2}}\left(X_{2}^{o}, e^{t D_{2}^{o}}\right) \in H F F_{*}(-Y,-\gamma)$. There is a natural pairing $H F F_{*}(Y, \gamma) \otimes$ $H F F_{*}(-Y,-\gamma) \rightarrow \mathbb{Q}[[t]]$, such that

$$
D_{X}^{(w, \Sigma)}\left(e^{t D}\right)=<\phi^{w_{1}}\left(X_{1}^{o}, e^{t D_{1}^{o}}\right), \phi^{w_{2}}\left(X_{2}^{o}, e^{t D_{2}^{o}}\right)>.
$$

When $b^{+}=1$, the invariants are calculated for a long neck, i.e. we refer to the invariants defined by $\Sigma$.

Proof.

- As explained in [2], the Floer homology groups $H F_{*}(Y)$ are well-defined since $\left.w\right|_{Y}$ is odd (this rules out problems with flat reducible connections on $Y)$. Also, there are invariants $\phi^{w_{1}}\left(X_{1}^{o},\left(D_{1}^{o}\right)^{n}\right) \in H F_{*}(Y), \phi^{w_{2}}\left(X_{2}^{o},\left(D_{2}^{o}\right)^{m}\right) \in$ $H F_{*}(-Y)$ such that

$$
D_{X}^{(w, \Sigma)}\left(\left(D_{1}^{o}\right)^{n}\left(D_{2}^{o}\right)^{m}\right)=<\phi^{w_{1}}\left(X_{1}^{o},\left(D_{1}^{o}\right)^{n}\right), \phi^{w_{2}}\left(X_{2}^{o},\left(D_{2}^{o}\right)^{m}\right)>.
$$

Now we write

$$
\phi^{w_{1}}\left(X_{1}^{o}, e^{t D_{1}^{o}}\right)=\sum \frac{t^{n}}{n !} \phi^{w_{1}}\left(X_{1}^{o},\left(D_{1}^{o}\right)^{n}\right)
$$

from where the statement of the theorem.

- Analogously, in [1] the Fukaya-Floer homology groups $H F F_{*}(Y, \gamma)$ are defined when $\left.w\right|_{Y}$ is odd. Associated to $\left(X_{i}^{o}, D_{i}^{o}\right)$, there are invariants $\phi^{w_{1}}\left(X_{1}^{o}, D_{1}^{o}\right) \in$ $H F F_{*}(Y, \gamma)$ and $\phi^{w_{2}}\left(X_{2}^{o}, D_{2}^{o}\right) \in H F F_{*}(-Y,-\gamma)$, where $\phi^{w_{1}}\left(X_{1}^{o}, D_{1}^{o}\right)$ is represented by a Fukaya-Floer chain $\left(\phi^{w_{1}}\left(X_{1}^{o},\left(D_{1}^{o}\right)^{n}\right)\right)_{n \geq 0}$, where $\phi^{w_{1}}\left(X_{1}^{o},\left(D_{1}^{o}\right)^{n}\right)$ $\in H F_{*}(Y)$ (and analogously for $\left(X_{2}^{o}, D_{2}^{o}\right)$ ), such that

$$
\left.D_{X}^{(w, \Sigma)}\left(D^{r}\right)=\sum_{0 \leq n \leq r}\left(\begin{array}{l}
r \\
n
\end{array}\right)<\phi^{w_{1}}\left(X_{1}^{o},\left(D_{1}^{o}\right)^{n}\right), \phi^{w_{2}}\left(X_{2}^{o},\left(D_{2}^{o}\right)^{r-n}\right)\right\rangle .
$$

So we write formally

$$
\phi^{w_{1}}\left(X_{1}^{o}, e^{t D_{1}^{o}}\right)=\sum \frac{t^{n}}{n !} \phi^{w_{1}}\left(X_{1}^{o},\left(D_{1}^{o}\right)^{n}\right)
$$

for the given Fukaya-Floer homology class. 
Now we particularize to the case which concerns us, $Y=\Sigma \times \mathbb{S}^{1}$. Conjugation in the second factor produces an isomorphism $Y \cong(-Y)$ (also $(Y, \gamma) \cong(-Y,-\gamma))$. As explained in [1] [17], $\operatorname{HFF}_{*}(Y, \gamma)$ is the limit of a spectral sequence whose $E_{3}$-term is $H F_{*}(Y) \otimes \hat{H}_{*}\left(\mathbb{C P}^{\infty}\right)$ (here $\hat{H}_{*}\left(\mathbb{C P}^{\infty}\right)$ means the natural completion of $H_{*}\left(\mathbb{C P}^{\infty}\right)$, i.e. $\mathbb{Q}[[t]], t$ of degree 2$)$, with differencital $d_{3}$ given by

$$
\mu(\gamma): H F_{i}(Y) \otimes H_{j}\left(\mathbb{C P}^{\infty}\right) \rightarrow H F_{i-3}(Y) \otimes H_{j+2}\left(\mathbb{C P}^{\infty}\right) .
$$

Let $\gamma=$ pt $\times \mathbb{S}^{1} \subset \Sigma \times \mathbb{S}^{1}$. Now all the differentials in the $E_{3}$ term of the spectral sequence are of the form (see Proposition 20) $H_{\text {odd }}\left(M_{\Sigma}^{\text {odd }}\right) \rightarrow H_{\text {even }}\left(M_{\Sigma}^{\text {odd }}\right)$ and $H_{\text {even }}\left(M_{\Sigma}^{\text {odd }}\right) \rightarrow H_{\text {odd }}\left(M_{\Sigma}^{\text {odd }}\right)$. The boundary cycle $\gamma=\mathbb{S}^{1}$ is invariant under the action of the group $\operatorname{Diff}(\Sigma)$ on $Y=\Sigma \times \mathbb{S}^{1}$, so the differentials commute with the action of $\operatorname{Diff}(\Sigma)$. As there are elements $\rho \in \operatorname{Diff}(\Sigma)$ acting as -1 on $H^{1}(\Sigma)$, we have that $\rho$ acts as -1 on $H_{\text {odd }}\left(M_{\Sigma}^{\text {odd }}\right)$ and as 1 on $H_{\text {even }}\left(M_{\Sigma}^{\text {odd }}\right)$. Therefore the differentials are zero and the spectral sequence degenerates in the third term. This implies that $H F F_{*}(Y, \gamma)=H F_{*}(Y) \otimes \hat{H}^{*}\left(\mathbb{C P}^{\infty}\right)=V[[t]]$, where $V=H F_{*}(Y)$. Now the relative invariants for $\left(X_{1}^{o}, D_{1}^{o}\right)$ can be written as

$$
\phi^{w_{1}}\left(X_{1}^{o}, e^{t D_{1}^{o}}\right)=\sum \frac{t^{n}}{n !} \phi^{w_{1}}\left(X_{1}^{o},\left(D_{1}^{o}\right)^{n}\right) \in V[[t]]
$$

where $\phi^{w_{1}}\left(X_{1}^{o},\left(D_{1}^{o}\right)^{n}\right) \in H F_{*}(Y)$ has perfect meaning. Under the isomorphism $H F F_{*}=H F F_{*}(Y, \gamma)=V[[t]], H F F_{*}$ becomes a $\mathbb{Q}[[t]]$-module and $H F F_{*} \otimes H F F_{*} \rightarrow$ $\mathbb{Q}[[t]]$ is $\mathbb{Q}[[t]]$-bilinear.

COROLlaRY 19.

1. There is a (rational) vector space $V=H F_{*}(Y)$ endowed with a bilinear form such that for every permissible $(X, \Sigma), w \in H^{2}\left(X^{o} ; \mathbb{Z}\right)$ with $\left.w\right|_{Y}$ an odd multiple of P.D. $\left[\mathbb{S}^{1}\right]$, and cycle $D^{o} \subset X^{o}$ with $\partial D^{o}=\emptyset$, we have $\phi^{w}\left(X^{o}, e^{t D^{\circ}}\right) \in$ $V[[t]]$. For $X=X_{1}^{o} \cup_{Y} X_{2}^{o}, D=D_{1}^{o}+D_{2}^{o}, \partial D_{1}^{o}=\partial D_{2}^{o}=\emptyset$, we have

$$
D_{X}^{(w, \Sigma)}\left(e^{t D}\right)=<\phi^{w_{1}}\left(X_{1}^{o}, e^{t D_{1}^{o}}\right), \phi^{w_{2}}\left(X_{2}^{o}, e^{t D_{2}^{o}}\right)>.
$$

2. There is a canonical isomorphism $H F F_{*}\left(Y, \mathbb{S}^{1}\right) \cong V[[t]]$, such that for every permissible $(X, \Sigma), w \in H^{2}\left(X^{o} ; \mathbb{Z}\right)$ with $\left.w\right|_{Y}$ an odd multiple of P.D.[ $\left.\mathbb{S}^{1}\right]$, and cycle $D^{o} \subset X^{o}$ with $\partial D^{o}=\mathbb{S}^{1}$, we have $\phi^{w}\left(X^{o}, e^{t D^{o}}\right) \in V[[t]]$. For $X=X_{1}^{o} \cup_{Y} X_{2}^{o}, D=D_{1}^{o}+D_{2}^{o}, \partial D_{1}^{o}=-\partial D_{2}^{o}=\mathbb{S}^{1}$, we have

$$
D_{X}^{(w, \Sigma)}\left(e^{t D}\right)=<\phi^{w_{1}}\left(X_{1}^{o}, e^{t D_{1}^{\circ}}\right), \phi^{w_{2}}\left(X_{2}^{o}, e^{t D_{2}^{o}}\right)>.
$$

Proposition 20. Let $M_{\Sigma}^{\text {odd }}$ be the moduli space of odd degree rank two stable vector bundles on $\Sigma$, which is a smooth variety [9]. Then there is an isomorphism

$$
H F_{*}(Y) \cong H_{*}\left(M_{\Sigma}^{\text {odd }}\right)
$$

as vector spaces (we are using rational coefficients), where we reduce the grading of $H_{*}\left(M_{\Sigma}^{\text {odd }}\right)$ modulo 4 .

Proof. Dostoglou and Salamon [5] prove $H F_{*}\left(\Sigma \times \mathbb{S}^{1}\right) \cong H F_{*}^{\text {symp }}\left(M_{\Sigma}^{\text {odd }}\right)$. It is the particular case where we consider $\phi=$ id $: \Sigma \rightarrow \Sigma$, in which the mapping torus of $\phi$ is $\Sigma \times \mathbb{S}^{1}$. As explained in the introduction of [5], the symplectic manifold $M_{\Sigma}^{\text {odd }}$ is connected, simply connected and $\pi_{2}\left(M_{\Sigma}^{\text {odd }}\right)=\mathbb{Z}$, so the groups $H F_{*}^{\text {symp }}\left(M_{\Sigma}^{\text {odd }}\right)$ are well-defined. Now $H F_{*}^{\text {symp }}\left(M_{\Sigma}^{\text {odd }}\right) \cong H_{*}\left(M_{\Sigma}^{\text {odd }}\right)$ is a standard result obtained by Floer himself [7] for proving the Arnold conjecture. 
There is a map

$$
\mu: H_{*}(\Sigma) \rightarrow H^{4-*}\left(M_{\Sigma}^{\text {odd }}\right)
$$

given by $\mu(\alpha)=-\frac{1}{4} p_{1}(\mathcal{V}) / 4$, where $\mathcal{V} \rightarrow \Sigma \times M_{\Sigma}^{\text {odd }}$ is the associated universal $S O(3)$ bundle, $p_{1}(\mathcal{V}) \in H^{4}\left(\Sigma \times M_{\Sigma}^{\text {odd }}\right)$ its first Pontrjagin class. Fix a basis $\left\{\gamma_{i}\right\}$ of $H_{1}(\Sigma)$. Let $a=\mu(\Sigma), b=\mu(x), c_{i}=\mu\left(\gamma_{i}\right)$. These elements generate $H^{*}\left(M_{\Sigma}^{\text {odd }}\right)$ as a ring [9] [21]. So there is a basis for $V=H^{*}\left(M_{\Sigma}^{\text {odd }}\right)$ with elements of the form

$$
f_{\alpha}=a^{n} b^{m} c_{i_{1}} \cdots c_{i_{r}} \in V
$$

for a finite set of indices of the form $\alpha=\left(n, m ; i_{1}, \ldots, i_{r}\right), n, m \geq 0, r \geq 0,1 \leq i_{1}<$ $\cdots<i_{r} \leq 2 g$. Let $N=\operatorname{dim} V$. We order the set of indices $\{\alpha\}$ so we identify such set with $\{1, \ldots, N\}$ and write $1 \leq \alpha \leq N$ in general.

Let $I$ be the ideal of $H^{*}\left(M_{\Sigma}^{\text {odd }}\right)$ generated by $c_{1}, \ldots, c_{2 g}$. Then the elements $a^{n} b^{m}$, $0 \leq n, m<g$, generate the quotient $H^{*}\left(M_{\Sigma}^{\text {odd }}\right) / I$ (see [9]). So we can suppose these elements are the first $g^{2}$ elements in the basis $\left\{f_{\alpha}\right\}$, i.e. for $1 \leq \alpha \leq g^{2}$.

The intersection pairing in $H^{*}\left(M_{\Sigma}^{\text {odd }}\right)$ is given by

$$
<f_{\alpha}, f_{\beta}>=<f_{\alpha} \cup f_{\beta},\left[M_{\Sigma}^{\text {odd }}\right]>\text {. }
$$

Therefore the intersection matrix $\left.\left(<f_{\alpha}, f_{\beta}\right\rangle\right)$ is invertible.

Here we recall that we have defined the manifold $A=\Sigma \times D^{2}$, with boundary $Y=\Sigma \times \mathbb{S}^{1}$, and let $\Delta=$ pt $\times D^{2} \subset A$ be the horizontal slice with $\partial \Delta=\mathbb{S}^{1}$. Put $w=$ P.D. $[\Delta] \in H^{2}(A ; \mathbb{Z})$. Put $\mathbb{A}(\Sigma)=\operatorname{Sym}^{*}\left(H_{0}(\Sigma) \oplus H_{2}(\Sigma)\right) \otimes \bigwedge^{*} H_{1}(\Sigma)$, so there is a natural map $\mathbb{A}(\Sigma) \rightarrow \mathbb{A}(X)$, whenever $\Sigma \hookrightarrow X$. Then we define

$$
\begin{aligned}
& z_{\alpha}=\Sigma^{n} x^{m} \gamma_{i_{1}} \cdots \gamma_{i_{r}} \in \mathbb{A}(\Sigma), \\
& e_{\alpha}=\phi^{w}\left(A, z_{\alpha} e^{t \Delta}\right) \in H F F_{*}\left(Y, \mathbb{S}^{1}\right)=V[[t]] .
\end{aligned}
$$

LEMMA 21. The intersection matrix $\left(<e_{\alpha}, e_{\beta}\right\rangle$ ) (with coefficients in $\left.\mathbb{Q}[[t]]\right)$ is invertible. Therefore, $\left\{e_{\alpha}\right\}$ is a basis for $H_{F} F_{*}(Y)=V[[t]]$.

Proof. As the elements $f_{\alpha} \in H^{*}\left(M_{\Sigma}^{\text {odd }}\right)$ have an integer degree between 0 and $6 g-6=\operatorname{dim} M_{\Sigma}^{\text {odd }}$, we can reorder the basis $\left\{f_{\alpha}\right\}$ such that the degree goes increasing (we use this special ordering only in this lemma). Now $z_{\alpha}=\Sigma^{n} x^{m} \gamma_{i_{1}} \cdots \gamma_{i_{r}}, z_{\beta}=$ $\Sigma^{n^{\prime}} x^{m^{\prime}} \gamma_{i_{1}^{\prime}} \cdots \gamma_{i_{r^{\prime}}}$ and

$$
<e_{\alpha}, e_{\beta}>=<\phi^{w}\left(A, z_{\alpha} e^{t \Delta}\right), \phi^{w}\left(A, z_{\beta} e^{t \Delta}\right)>=D_{\Sigma \times \mathbb{C P}^{1}}^{(w, \Sigma)}\left(z_{\alpha} z_{\beta} e^{t \mathbb{C P}^{1}}\right)
$$

where $w=$ P.D. $\left[\mathbb{C P}^{1}\right] \in H^{2}\left(\Sigma \times \mathbb{C P}^{1} ; \mathbb{Z}\right)$. The matrix $\left(<e_{\alpha}, e_{\beta}>\right)$ has coefficients in $\mathbb{Q}[[t]]$, so it is invertible if and only if its determinant is a unit in $\mathbb{Q}[[t]]$, i.e. when we put $t=0$ we obtain an invertible matrix with rational coefficients. Now

$$
<e_{\alpha}, e_{\beta}>\left.\right|_{t=0}=D_{\Sigma \times \mathbb{C P}^{1}}^{(w, \Sigma)}\left(z_{\alpha} z_{\beta}\right) .
$$

The lowest dimension of the moduli spaces of anti-self-dual connections for $\Sigma \times \mathbb{C P}^{1}$ is $6 g-6$, so if $\operatorname{deg} z_{\alpha}+\operatorname{deg} z_{\beta}<6 g-6$ then $<e_{\alpha}, e_{\beta}>\left.\right|_{t=0}=0$. The moduli space of dimension $6 g-6$ corresponds to $p_{1}=0$ and $w=$ P.D. $\left[\mathbb{C P}^{1}\right]$. All of these connections are flat and irreducible, actually pull-back of flat connections on $\Sigma$ with $\left.w\right|_{\Sigma}$ odd, so 
the corresponding moduli space is isomorphic to $M_{\Sigma}^{\text {odd }}$. Thus if $\operatorname{deg} z_{\alpha}+\operatorname{deg} z_{\beta}=6 g-6$ then $<e_{\alpha}, e_{\beta}>\left.\right|_{t=0}=<f_{\alpha}, f_{\beta}>$. The matrix $\left(<f_{\alpha}, f_{\beta}>\right)$ is of the form

$$
\left(\begin{array}{ccccc}
0 & 0 & \cdots & 0 & A_{0} \\
0 & 0 & \cdots & A_{1} & 0 \\
\vdots & \vdots & \ddots & \vdots & \vdots \\
A_{6 g-6} & 0 & \cdots & 0 & 0
\end{array}\right)
$$

where $A_{i}$ are submatrices corresponding to the intersection product

$$
H^{i}\left(M_{\Sigma}^{\text {odd }}\right) \otimes H^{6 g-6-i}\left(M_{\Sigma}^{\text {odd }}\right) \rightarrow \mathbb{Q}
$$

So all $A_{i}$ have non-vanishing $\operatorname{determinant} \operatorname{det} A_{i} \in \mathbb{Q}$. Finally, we have that the matrix $\left(<e_{\alpha}, e_{\beta}>\left.\right|_{t=0}\right)$ is

$$
\left(\begin{array}{ccccc}
0 & 0 & \cdots & 0 & A_{0} \\
0 & 0 & \cdots & A_{1} & * \\
\vdots & \vdots & \ddots & \vdots & \vdots \\
A_{6 g-6} & * & \cdots & * & *
\end{array}\right)
$$

and it is invertible.

3. Proof of Theorem 9. By the above lemma, $\left\{e_{\alpha}\right\}$ is a basis of $V[[t]]$, so there is an isomorphism

$$
\begin{aligned}
V[[t]] & \rightarrow \mathbb{Q}^{N}[[t]] \\
\phi & \mapsto\left(<\phi, e_{\alpha}>\right)_{\alpha} .
\end{aligned}
$$

The important feature is that if $\left(X_{1}, \Sigma_{1}\right)$ is permissible, $w \in H^{2}\left(X_{1} ; \mathbb{Z}\right)$ with $w \cdot \Sigma \equiv 1$ $(\bmod 2), D_{1}^{o} \subset X_{1}^{o}$ with $\partial D_{1}^{o}=\mathbb{S}^{1}, D_{1}=D_{1}^{o}+\Delta$, then $\phi=\phi^{w}\left(X_{1}^{o}, e^{t D_{1}^{o}}\right)$ goes to $\left(c_{X_{1}, \alpha}(t)\right)_{1 \leq \alpha \leq N} \in \mathbb{Q}^{N}[[t]]$, where

$$
c_{X_{1}, \alpha}(t)=D_{X_{1}}^{(w, \Sigma)}\left(z_{\alpha} e^{t D_{1}}\right)
$$

The pairing in $V[[t]]$ corresponds through the isomorphism to a pairing in $\mathbb{Q}^{N}[[t]]$, which is $\mathbb{Q}[[t]]$-bilinear, hence given by a matrix of $\mathbb{Q}[[t]]$-coefficients $\left(M_{\alpha \beta}(t)\right)_{1 \leq \alpha, \beta \leq N}$. This matrix is universal (only dependent on the data necessary for the construction of the Fukaya-Floer groups, i.e. $(Y, \gamma)$, and on the chosen basis).

Now if $\left(X_{1}, \Sigma_{1}\right)$ and $\left(X_{2}, \Sigma_{2}\right)$ are permissible, let $X=X(\phi)=X_{1} \#_{\Sigma} X_{2}$ (with an identification $\phi$ ) be the connected sum along $\Sigma$, with the induded homology orientation. Let $D \in H_{2}(X)$ with $D=D_{1}^{o}+D_{2}^{o}, \partial D_{1}^{o}=-\partial D_{2}^{o}=\mathbb{S}^{1}$. Put $D_{i}=D_{i}^{o}+\Delta$. Then (here $\left.c_{X_{1}, \alpha}(t)=D_{X_{1}}^{(w, \Sigma)}\left(z_{\alpha} e^{t D_{1}}\right), c_{X_{2}, \beta}(t)=D_{X_{2}}^{(w, \Sigma)}\left(z_{\beta} e^{t D_{2}}\right)\right)$,

(6) $D_{X}^{(w, \Sigma)}\left(e^{t D}\right)=<\phi^{w}\left(X_{1}^{o}, e^{t D_{1}^{o}}\right), \phi^{w}\left(X_{2}^{o}, e^{t D_{2}^{o}}\right)>=\sum_{1 \leq \alpha, \beta \leq N} c_{X_{1}, \alpha}(t) M_{\alpha \beta}(t) c_{X_{2}, \beta}(t)$

Now suppose that $X_{1}$ has $b_{1}=0$. Then $c_{X_{1}, \alpha}(t)=0$ whenever $r>0$ (recall $\left.\alpha=\left(n, m ; i_{1}, \ldots, i_{r}\right)\right)$. So the only non-zero coordinates correspond to $z_{\alpha}=\Sigma^{n} x^{m}$, $0 \leq n, m \leq g-1$. Suppose furthermore $X_{1}$ of simple type with $b_{1}=0$ and $b^{+}>1$, so for $z=\left(x^{2}-4\right) \Sigma^{n} x^{m-2}, 0 \leq n \leq g-1,2 \leq m \leq g-1, D_{X_{1}}^{(w, \Sigma)}\left(z e^{t D_{1}}\right)=0$, hence 
changing the basis $\left\{z_{\alpha}\right\}$, all coordinates $c_{X_{1}, \alpha}(t)$ are zero except for the first $2 g$ of them, corresponding to $z_{\alpha}=\Sigma^{n}$ and $z_{\alpha}=\Sigma^{n} x, 0 \leq n \leq g-1$.

LemMA 22. Let $\left(X_{1}, \Sigma_{1}\right)$ be permissible, with $\Sigma_{1}$ of genus $g, X_{1}$ of simple type with $b_{1}=0$ and $b^{+}>1,(w, \Sigma)$ allowable and $D_{1} \in H_{2}\left(X_{1}\right)$ with $D_{1} \cdot \Sigma=1$. Then $D_{X_{1}}^{(w, \Sigma)}\left(e^{t D_{1}} z\right)=0$ for

$z= \begin{cases}\left(1-\frac{x}{2}\right)(\Sigma+t)\left((\Sigma+t)^{2}+4^{2}\right)\left((\Sigma+t)^{2}+8^{2}\right) \cdots\left((\Sigma+t)^{2}+(2 g-4)^{2}\right) g \text { even } \\ \left(1+\frac{x}{2}\right)\left((\Sigma-t)^{2}-2^{2}\right)\left((\Sigma-t)^{2}-6^{2}\right) \cdots\left((\Sigma-t)^{2}-(2 g-4)^{2}\right) & g \text { odd }\end{cases}$

Proof. Suppose, for instance, $g$ even. By remark 4 , for any polynomial $p(\Sigma)$ in $\Sigma$ (with coefficients in $\mathbb{Q}[[t]]$ ),

$$
D_{X_{1}}^{(w, \Sigma)}\left(\left(1-\frac{x}{2}\right) p(\Sigma) e^{t D_{1}}\right)=2 e^{-Q\left(t D_{1}\right) / 2} \sum_{K_{j} \cdot \Sigma \equiv 0} i^{-d_{0}} p\left(\left(-t D_{1}+i K_{j}\right) \cdot \Sigma\right) a_{j, w} e^{i t K_{j} \cdot D_{1}}
$$

Now $D_{1} \cdot \Sigma=1$, so the expression above vanishes when $p(\Sigma)$ has roots $-t,-t \pm 4 i, \ldots-$ $t \pm(2 g-4) i$.

Note that $z=\left(1 \pm \frac{x}{2}\right) p(\Sigma)$ with $p$ of degree $g-1$. Let us choose a basis $\left\{z_{\alpha}\right\}$ with $z_{1}, \ldots z_{2 g-1}$ being the elements

$$
1, \Sigma, \Sigma^{2}, \ldots, \Sigma^{g-1}, x, \Sigma x, \ldots, \Sigma^{g-2} x
$$

$z_{2 g}=z$, also $z_{2 g+1}, \ldots, z_{g^{2}}$ being the elements $\left(x^{2}-4\right) \Sigma^{n} x^{m-2}, 0 \leq n \leq g-1$, $2 \leq m \leq g-1$ and $e_{g^{2}+1}, \ldots, e_{N}$ having all $r>0$. So, when $X_{1}$ is of simple type with $b_{1}=0$ and $b^{+}>1$,

$$
\phi^{w}\left(X_{1}^{o}, e^{t D_{1}^{o}}\right) \in \mathbb{Q}^{2 g-1}[[t]] \subset V[[t]]
$$

where $\mathbb{Q}^{2 g-1}[[t]]$ is the orthogonal complement to $\left\langle e_{2 g}, \ldots, e_{N}>\right.$ in $V[[t]]$.

Formula (6) reduces to (when both $X_{i}$ are of simple type with $b_{1}=0$ and $b^{+}>1$ )

$$
D_{X}^{(w, \Sigma)}\left(e^{t D}\right)=\sum_{1 \leq \alpha, \beta \leq 2 g-1} c_{X_{1}, \alpha}(t) M_{\alpha \beta}(t) c_{X_{2}, \beta}(t)
$$

where

$$
\begin{aligned}
& c_{X_{1}, \alpha}(t)= \begin{cases}D_{X_{1}}^{(w, \Sigma)}\left(\Sigma^{n} e^{t D_{1}}\right) & \text { if } 0 \leq n \leq g-1, \alpha=(n, 0 ;)=n+1 \\
D_{X_{1}}^{(w, \Sigma)}\left(\Sigma^{n} x e^{t D_{1}}\right) & \text { if } 0 \leq n \leq g-2, \alpha=(n, 1 ;)=n+g+1\end{cases} \\
& =\left\{\begin{array}{c}
e^{Q\left(t D_{1}\right) / 2} \sum a_{j, w}\left(1+K_{j} \cdot \Sigma\right)^{n} e^{t K_{j} \cdot D_{1}}+ \\
K_{j} \cdot \Sigma \equiv 2 \quad(\bmod 4) \\
+e^{-Q\left(t D_{1}\right) / 2} \sum i^{-d_{0}} a_{j, w}\left(-1+i K_{j} \cdot \Sigma\right)^{n} e^{t i K_{j} \cdot D_{1}} \\
K_{j} \cdot \Sigma \equiv 0(\bmod 4) \\
2 e^{Q\left(t D_{1}\right) / 2} \sum a_{j, w}\left(1+K_{j} \cdot \Sigma\right)^{n} e^{t K_{j} \cdot D_{1}}- \\
K_{j} \cdot \Sigma \equiv 2 \quad(\bmod 4) \\
-2 e^{-Q\left(t D_{1}\right) / 2} \sum i^{-d_{0}} a_{j, w}\left(-1+i K_{j} \cdot \Sigma\right)^{n} e^{t i K_{j} \cdot D_{1}} \\
K_{j} \cdot \Sigma \equiv 0(\bmod 4)
\end{array}\right.
\end{aligned}
$$

and analogously for $c_{X_{2}, \beta}(t)$. 
So it is easy to find another basis $\left\{z_{1}, \ldots, z_{2 g-1}\right\}$ (which we do not write explicitly) spanning $\mathbb{Q}^{2 g-1}$ such that

$$
c_{X_{1}, \alpha}(t)= \begin{cases}e^{Q\left(t D_{1}\right) / 2} \sum_{K_{j} \cdot \Sigma=2 p} a_{j, w} e^{t K_{j} \cdot D_{1}} & \text { if } p \text { is odd } \\ e^{-Q\left(t D_{1}\right) / 2} \sum_{K_{j} \cdot \Sigma=2 p} i^{-d_{0}} a_{j, w} e^{t i K_{j} \cdot D_{1}} & \text { if } p \text { is even }\end{cases}
$$

where $\alpha=1, \ldots, 2 g-1$ corresponds to $p=g-1,-(g-1), g-2,-(g-2), \ldots, 0$. Formula (7) yields

$$
D_{X}^{(w, \Sigma)}\left(e^{t D}\right)=\sum_{1 \leq \alpha, \beta \leq 2 g-1} c_{X_{1}, \alpha}(t) M_{\alpha \beta}(t) c_{X_{2}, \beta}(t)
$$

with $c_{X_{2}, \beta}(t)$ defined analogously to $c_{X_{1}, \alpha}(t)$, with the letter $q$ in the place of $p$.

LEMMA 23. The matrix $\left(M_{\alpha \beta}(t)\right)$ is diagonal.

Proof. First we note that

$$
D_{X}^{(w, \Sigma)}\left(e^{t D+s \Sigma}\right)=\sum_{p \text { odd }} c_{X, \alpha}(t) e^{t s+2 p s}+\sum_{p \text { even }} c_{X, \alpha}(t) e^{-t s+2 p i s}
$$

We use equation (8) for $D+\frac{s}{t} \Sigma, D_{1}+\frac{s}{t} \Sigma$ and $D_{2}$, so

$$
\begin{aligned}
D_{X}^{(w, \Sigma)}\left(e^{t D+s \Sigma}\right)= & \sum_{\substack{1 \leq \alpha, \beta \leq 2 g-1 \\
p \text { odd }}} c_{X_{1}, \alpha}(t) M_{\alpha \beta}(t) c_{X_{2}, \beta}(t) e^{t s+2 p s} \\
& +\sum_{\substack{1 \leq \alpha, \beta \leq 2 g-1 \\
p \text { odd }}} c_{X_{1}, \alpha}(t) M_{\alpha \beta}(t) c_{X_{2}, \beta}(t) e^{-t s+2 p i s}
\end{aligned}
$$

Then

$$
c_{X, \alpha}(t)=\sum_{\beta} c_{X_{1}, \alpha}(t) M_{\alpha \beta}(t) c_{X_{2}, \beta}(t)
$$

Let us see that $M_{\alpha \beta}(t)=0$ unless $\beta=\alpha$. Suppose, for instance, that $p$ is odd. If we write now $D=\left(D_{1}^{o}+r \Sigma\right)+\left(D_{2}^{o}-r \Sigma\right)$, the left hand side of (9) remains unchanged, but the right hand side is a sum of exponentials $e^{(2 p-2 q) r}, q$ odd, and $e^{(2 p-2 q i) r}, q$ even. So when $q \neq p$, it is $M_{\alpha \beta}(t)=0$.

Formula (8) gives

$$
\begin{aligned}
& D_{X}^{(w, \Sigma)}\left(e^{t D}\right)= \sum_{K_{j} \cdot \Sigma=L_{k} \cdot \Sigma=2 p, p \text { odd }} e^{Q\left(t D_{1}\right) / 2+Q\left(t D_{2}\right) / 2} M_{\alpha \alpha}(t) a_{j, w} b_{k, w} e^{t K_{j} \cdot D_{1}+t L_{k} \cdot D_{2}}+ \\
&+\sum_{K_{j} \cdot \Sigma=L_{k} \cdot \Sigma=2 p, p \text { even }}\left\{e^{-Q\left(t D_{1}\right) / 2-Q\left(t D_{2}\right) / 2} M_{\alpha \alpha}(t) i^{-d_{0}\left(X_{1}, w_{1}\right)-d_{0}\left(X_{2}, w_{2}\right)}\right. \\
&\left.a_{j, w} b_{k, w} e^{t i K_{j} \cdot D_{1}+t i L_{k} \cdot D_{2}}\right\} .
\end{aligned}
$$

Obviously $D^{2}=D_{1}^{2}+D_{2}^{2}$. We are assuming that $X$ is of simple type and recall that $b_{1}(X)=0$ and $b^{+}(X)>1$. By remark $11, d_{0}(X, w)-d_{0}\left(X_{1}, w_{1}\right)-d_{0}\left(X_{2}, w_{2}\right)=$ $w^{2}-w_{1}^{2}-w_{2}^{2}-3(g-1) \equiv g-1 \quad(\bmod 4)$ (since we are assuming $w^{2} \equiv w_{1}^{2}+w_{2}^{2}$ $(\bmod 4))$, so

$$
\mathbb{D}_{X}^{w}\left(e^{t D}\right)=e^{Q(t D) / 2} \sum_{\substack{K_{j} \cdot \Sigma=L_{k} \cdot \Sigma=2 p \\-(g-1) \leq p \leq g-1}} i^{s} M_{\alpha \alpha}(t) a_{j, w} b_{k, w} e^{t K_{j} \cdot D_{1}+t L_{k} \cdot D_{2}}
$$


where $s=0$ when $p$ is odd and $s=g-1$ when $p$ is even. As $g$ is not dependent on the particular manifolds, we absorb this factor into the matrix without affecting its universality.

This expression is valid for any $D \in H_{2}(X)$ such that $\left.D\right|_{Y}=\mathbb{S}^{1}$. We note that it does not change if we change $\left(D_{1}, D_{2}\right)$ for $\left(D_{1}+r \Sigma, D_{2}-r \Sigma\right)$, as expected. This means that we only need to assume the conditions: $D$ and $D_{i}$ coincide in $X_{i}^{o}$, $i=1,2$, and $D^{2}=D_{1}^{2}+D_{2}^{2}$. Now take a linear map $D \mapsto\left(D_{1}, D_{2}\right)$ from the subspace $\mathcal{H}=\left\{D \in H_{2}(X) /\left.D\right|_{Y}=k\left[\mathbb{S}^{1}\right]\right.$, some $\left.k\right\}$, satisfying the former conditions. As the set of $D \in H_{2}(X)$ with $\left.D\right|_{Y}$ a non-zero multiple of $\mathbb{S}^{1}$ is dense in $\mathcal{H}$, we have that for any $D \in \mathcal{H}$,

$$
\mathbb{D}_{X}^{w}\left(e^{t D}\right)=e^{Q(t D) / 2} \sum_{K_{j} \cdot \Sigma=L_{k} \cdot \Sigma=2 p} M_{\alpha \alpha}(t(D \cdot \Sigma)) a_{j, w} b_{k, w} e^{t K_{j} \cdot D_{1}+t L_{k} \cdot D_{2}}
$$

LEMMA 24. $M_{\alpha \alpha}(t)=0$, except for $\alpha=1,2$.

Proof. Let $Y$ be the $K 3$ surface, which is a manifold of simple type with $b_{1}=0$ and $b^{+}>1$ (see [12]). Consider a tight embedded Riemann surface $\Sigma^{\prime} \hookrightarrow Y$ of genus $g^{\prime}<g$ and self-intersection zero. By definition of tightness (see [12]), $\left(\Sigma^{\prime}\right)^{2}=2 g^{\prime}-2$. To construct it, we consider an elliptic fibration for the $K 3$ surface. Let $T$ be a generic fibre (which is a torus of self-intersection zero) and let $S$ be a section represented by a sphere of self-intersection -2 . Then consider $S$ together with $g^{\prime}$ generic fibres and smooth out the intersection points. Call the resulting Riemann surface $\Sigma^{\prime}$. It is homologous to $S+g^{\prime} T$, it has genus $g^{\prime}$ and self-intersection $2 g^{\prime}-2$, as required.

We blow-up $Y$ at $2 g^{\prime}-2$ points in $\Sigma^{\prime}$, to get $X_{1}=Y \#\left(2 g^{\prime}-2\right) \overline{\mathbb{C P}}^{2}$. The proper transform of $\Sigma^{\prime}$ is a Riemann surface of genus $g^{\prime}$ and self-intersection zero. Perform an internal connected sum with $g-g^{\prime}$ homologically trivial tori to obtain a Riemann surface $\Sigma_{1}$ of genus $g$ and self-intersection zero. If $E_{1}, \ldots, E_{2 g^{\prime}-2}$ are the exceptional divisors, $\Sigma_{1}$ is homologous to $\Sigma^{\prime}+E_{1}+\cdots+E_{2 g^{\prime}-2}$.

Moreover, the basic classes of $X_{1}$ are $\pm E_{1}+\cdots \pm E_{2 g^{\prime}-2}$. They all satisfy $\kappa \cdot \Sigma_{1} \leq$ $2 g^{\prime}-2$ and there is exactly one, $K=E_{1}+\cdots+E_{2 g^{\prime}-2}$, satisfying the equality.

Let $\left(X_{2}, \Sigma_{2}\right)=\left(X_{1}, \Sigma_{1}\right)$, and consider $X=X_{1} \#_{\Sigma} X_{2}$ with the preferred identification of remark 7 (double of $X_{1}$ along $\Sigma_{1}$ ). Then $X$ splits off a $\mathbb{S}^{2} \times \mathbb{S}^{2}$, so its invariants are zero (see [16, section 4.3] for this well-known phenomenon). As in the proof of lemma $23, c_{X, \alpha}(t)=c_{X_{1}, \alpha}(t) M_{\alpha \alpha}(t) c_{X_{2}, \alpha}(t)$. We proceed by induction from $g^{\prime}=1,2, \ldots, g-1$ and get $M_{\alpha \alpha}(t)=0$, for $\alpha \geq 3$.

Formula (10) becomes

$$
\begin{gathered}
\mathbb{D}_{X}^{w}\left(e^{t D}\right)=e^{Q(t D) / 2} \sum_{K_{j} \cdot \Sigma=L_{k} \cdot \Sigma=2 g-2} M_{11}(t(D \cdot \Sigma)) a_{j, w} b_{k, w} e^{t K_{j} \cdot D_{1}+t L_{k} \cdot D_{2}}+ \\
+e^{Q(t D) / 2} \sum_{K_{j} \cdot \Sigma=L_{k} \cdot \Sigma=-(2 g-2)} M_{22}(t(D \cdot \Sigma)) a_{j, w} b_{j, w} e^{t K_{j} \cdot D_{1}+t L_{k} \cdot D_{2}}
\end{gathered}
$$

Now let us compute $M_{11}(t)$ and $M_{22}(t)$. We can do that with a particular example of connected sum along a surface of genus $g$ in which the invariants are known for $X_{1}$, $X_{2}$ and $X=X_{1} \#_{\Sigma} X_{2}$.

DEFINITION 25. We are going to define the following smooth manifolds:

- Let $S_{n}$ be the minimal elliptic surface with no multiple fibres and geometric genus $p_{g}=n-1$. This is unique up to diffeomorphism [8]. $S_{1}$ is the rational elliptic surface, i.e. $S_{1}=\mathbb{C P}^{2} \# 9 \overline{\mathbb{C P}}^{2}$. 
- Let $\tilde{S}_{1}$ be the blow-up of $S_{1}$ at one point. Therefore, $\tilde{S}_{1}=\mathbb{C P}^{2} \# 10 \overline{\mathbb{C P}}^{2}$. Consider a particular elliptic fibration for $S_{1}$ with section $\sigma$ (of self-intersection $-1)$ and fibre $F$. Call $E$ the exceptional divisor, so $\tilde{S}_{1}$ has an elliptic $f$ bration with fibre the torus $T_{1}=F$ and there is another embedded torus $T_{2}$ homologous to $\sigma+T_{1}-E$ with $T_{2}^{2}=0$ and $T_{1} \cdot T_{2}=1$.

- Let $B_{g}=\underbrace{\tilde{S}_{1} \#_{T_{1}} \tilde{S}_{1} \#_{T_{1}} \cdots \#_{T_{1}} \tilde{S}_{1}}_{g}$ (connected sums along $T_{1}$ 's with the preferred identification), which is diffeomorphic to $S_{g} \# g \overline{\mathbb{C P}}^{2}$. It contains an embedded torus $T_{1}$ of self-intersection zero and a Riemann surface of genus $g$ (and self-intersection zero) made up by gluing smoothly the $T_{2}$ 's coming from each $\tilde{S}_{1}$. Actually, the elliptic surface $S_{g}$ has a section $\sigma_{g}$ with $\sigma_{g}^{2}=-g$, and $\Sigma_{g}$ can be taken to be the proper transform of $\sigma_{g}$. Clearly, $\Sigma_{g} \cdot T_{1}=1$, so $\left(B_{g}, \Sigma_{g}\right)$ is permissible. $B_{g}$ is of simple type with $b_{1}=0, b^{+}>1($ as $g \geq 2)$.

- Let $C_{g}=B_{g} \#_{\Sigma_{g}} B_{g}$ with the preferred identification. It contains a Riemann surface $\hat{\Sigma}_{2}$ of genus 2 and self-intersection zero made up from gluing smoothly the $T_{1}$ 's. If we perform instead the connected sum of two $\tilde{S}_{1}$ along $T_{2}$, we get $\hat{B}_{2}=\tilde{S}_{1} \# T_{2} \tilde{S}_{1}$ with an embedded Riemann surface $\hat{\Sigma}_{2}$ of genus 2 and selfintersection zero, coming from smoothly gluing the $T_{1}$ 's. Clearly $\left(\hat{B}_{2}, \hat{\Sigma}_{2}\right) \cong$ $\left(B_{2}, \Sigma_{2}\right)$. Now

$$
C_{g}=B_{g} \# \Sigma_{g} B_{g}=\underbrace{\hat{B}_{2} \#_{\hat{\Sigma}_{2}} \cdots \#_{\hat{\Sigma}_{2}} \hat{B}_{2}}_{g} .
$$

By [18, theorem 10], $C_{g}$ is of simple type with $b_{1}=0$ and $b^{+}>1 . A l$ ternatively, we can use [12], since it contains a torus of self-intersection 0 intersecting an embedded (-2)-sphere transversely in one point (see proposition 27).

Proposition 26. Consider $\left(B_{g}, \Sigma_{g}\right)$. Let $K_{B_{g}}$ be the canonical class of $B_{g}$, and $w=$ P.D. $\left[T_{1}\right] \in H^{2}\left(B_{g} ; \mathbb{Z}\right)$. Then $\mathbb{D}_{B_{g}}^{w}\left(e^{\bullet}\right)=\frac{1}{2^{2 g-2}} e^{Q / 2} e^{K_{B_{g}}}+\frac{1}{2^{2 g-2}} e^{Q / 2} e^{-K_{B_{g}}}+\cdots$, where the dots correspond to basic classes $\kappa$ for $B_{g}$ with $\left|\kappa \cdot \Sigma_{g}\right|<2 g-2$.

Proof. Write $B_{g}$ as $S_{g} \# g \overline{\mathbb{C P}}^{2}$. Let $F$ be the fibre of the natural elliptic fibration (i.e. $F=T_{1}$ ). Let $E_{1}, \ldots, E_{g}$ be the exceptional divisors. Then the basic classes are $k F \pm E_{1} \pm E_{2} \cdots \pm E_{g}$, with $-(g-2) \leq k \leq(g-2)$ and $k \equiv g-2 \quad(\bmod 2)$ (see [12] [6]). So the only basic class $\kappa$ with $\kappa \cdot \Sigma_{g}=2 g-2$ is the canonical class $K_{B_{g}}=(g-2) F+E_{1}+E_{2}+\cdots+E_{g}$. Therefore [12] [6] [17]

$$
\begin{aligned}
\mathbb{D}_{B_{g}}\left(e^{\bullet}\right) & =e^{Q / 2}(\sinh F)^{g-2} \sinh E_{1} \cdots \sinh E_{g} \\
& =\frac{1}{2^{2 g-2}} e^{Q / 2} e^{K_{B_{g}}}+\frac{1}{2^{2 g-2}} e^{Q / 2} e^{-K_{B_{g}}}+\cdots
\end{aligned}
$$

Now we note from proposition 1 that for $w=T_{1}, \mathbb{D}_{B_{g}}^{w}=\mathbb{D}_{B_{g}}$, since $w \cdot \kappa=0$ for all basic classes $\kappa$, hence the result.

Proposition 27. $C_{g}$ is of simple type. For $w=$ P.D. $\left[\hat{\Sigma}_{2}\right] \in H^{2}\left(C_{g} ; \mathbb{Z}\right)$,

$$
\mathbb{D}_{C_{g}}^{w}\left(e^{\alpha}\right)=-2^{3 g-5} e^{Q(\alpha) / 2} e^{K_{C_{g}} \cdot \alpha}+(-1)^{g} 2^{3 g-5} e^{Q(\alpha) / 2} e^{K_{C_{g}} \cdot \alpha},
$$

for a unique basic class $K=K_{C_{g}} \in H^{2}\left(C_{g} ; \mathbb{Z}\right)$, such that $K \cdot \hat{\Sigma}_{2}=2, K \cdot \Sigma_{g}=2 g-2$.

Proof. By propositon 26 and proposition 3,

$$
D_{\hat{B}_{2}}^{\left(T_{2}, \hat{\Sigma}_{2}\right)}\left(e^{\alpha}\right)=\frac{1}{4} e^{Q(\alpha) / 2} e^{K_{\hat{B}_{2}} \cdot \alpha}+\frac{1}{4} e^{Q(\alpha) / 2} e^{-K_{\hat{B}_{2}} \cdot \alpha}+\cdots
$$


where the dots correspond to basic classes $\kappa$ with $\kappa \cdot \hat{\Sigma}_{2}=0$. Now we express $C_{g}=$ $\underbrace{\hat{B}_{2} \#_{\hat{\Sigma}_{2}} \cdots \#_{\hat{\Sigma}_{2}} \hat{B}_{2}}_{g}$ and use $[18$, theorem 6$]$,

$$
\begin{aligned}
D_{C_{g}}^{\left(\Sigma_{g}, \hat{\Sigma}_{2}\right)}\left(e^{\alpha}\right) & =(-32)^{g-1}\left(\frac{1}{4}\right)^{g} e^{Q(\alpha) / 2} e^{K_{C_{g}} \cdot \alpha}+32^{g-1}\left(\frac{1}{4}\right)^{g} e^{Q(\alpha) / 2} e^{K_{C_{g}} \cdot \alpha}= \\
& =(-1)^{g-1} 2^{3 g-5} e^{Q(\alpha) / 2} e^{K_{C_{g}} \cdot \alpha}+2^{3 g-5} e^{Q(\alpha) / 2} e^{K_{C_{g}} \cdot \alpha},
\end{aligned}
$$

where $K=K_{C_{g}} \in H^{2}\left(C_{g} ; \mathbb{Z}\right)$ is defined as the unique cohomology class such that

- $K \cdot \hat{\Sigma}_{2}=2$

- $K \cdot \Sigma_{g}=2 g-2$

- Writing $C_{g}=C_{g-1}^{o} \cup_{Y} \hat{B}_{2}^{o}$, one has $K \cdot\left(\alpha_{1}+\alpha_{2}\right)=K_{C_{g-1}} \cdot \alpha_{1}+K_{\hat{B}_{2}} \cdot \alpha_{2}$, where $\alpha_{1} \in H_{2}\left(C_{g-1}^{o}\right)$ and $\alpha_{2} \in H_{2}\left(\hat{B}_{2}^{o}\right)$.

- Write $\hat{B}_{2}^{o}=\left(\tilde{S}_{1}-\left(N_{T_{1}} \cup N_{T_{2}}\right)\right) \cup Y-N_{T_{1}} \cap \partial N_{T_{2}}\left(\tilde{S}_{1}-\left(N_{T_{1}} \cup N_{T_{2}}\right)\right)$. For any $\gamma \subset \Sigma \subset \Sigma \times \mathbb{S}^{1}=\partial N_{T_{1}}$ disjoint from $N_{T_{2}}$, we consider the vanishing discs for $\tilde{S}_{1}$ with elliptic fibration with fibre $T_{1}$ (see [8, page 167]). These can be considered disjoint from $N_{T_{2}}$, so they are embedded (-1)-discs in $\hat{B}_{2}^{o}$ with boundary included in $\partial \hat{B}_{2}^{o}$. In the same vein we find many embedded (-1)discs in $C_{g-1}^{o}$, so, in the terminology of [14], the preferred identification for $C_{g}=C_{g-1}^{o} \cup_{Y} \hat{B}_{2}^{o}$ is admissible. By remark 14 , in the first place $C_{g}$ is of simple type. In the second place, there is a subspace $V \subset H_{2}(X)$ such that $H_{2}(X)=\mathcal{H} \oplus V$ and $K \cdot \alpha=0$, for all $\alpha \in V$.

For $w=$ P.D. $\left[\hat{\Sigma}_{2}\right]$, using proposition 3 again,

$$
\mathbb{D}_{C_{g}}^{\hat{\Sigma}_{2}}\left(e^{\alpha}\right)=-2^{3 g-5} e^{Q(\alpha) / 2} e^{K_{C_{g}} \cdot \alpha}+(-1)^{g} 2^{3 g-5} e^{Q(\alpha) / 2} e^{K_{C_{g}} \cdot \alpha}
$$

Finally, we can find $M_{11}(t)$ and $M_{22}(t)$. Let $\left(X_{1}, \Sigma_{1}\right)=\left(X_{2}, \Sigma_{2}\right)=\left(B_{g}, \Sigma_{g}\right)$, $w_{1}=w_{2}=$ P.D. $\left[T_{2}\right], D_{1}=D_{2}=T_{2}$ and $X=C_{g}, w=$ P.D. $\left[\hat{\Sigma}_{2}\right], D=\hat{\Sigma}_{2}, \Sigma=\Sigma_{g}$, so formula (11) and proposition 26 yield

$$
\mathbb{D}_{X}^{w}\left(e^{t D+s \Sigma}\right)=e^{t s}\left(M_{11}(t) \frac{1}{2^{2 g-2}} \frac{1}{2^{2 g-2}} e^{(2 g-2) s}+M_{22}(t) \frac{1}{2^{2 g-2}} \frac{1}{2^{2 g-2}} e^{-(2 g-2) s}\right)
$$

and use proposition 27 to get

$$
\mathbb{D}_{X}^{w}\left(e^{t D+s \Sigma}\right)=e^{t s}\left(-2^{3 g-5} e^{2 t+(2 g-2) s}+(-1)^{g} 2^{3 g-5} e^{-2 t-(2 g-2) s}\right),
$$

from where $M_{11}(t)=-2^{7 g-9} e^{2 t}, M_{22}(t)=(-1)^{g} 2^{7 g-9} e^{-2 t}$. This finishes the proof of theorem 9 .

4. Proof of Theorem 17. Let $S=\Sigma \times \mathbb{C P}^{1}, w=$ P.D. $\left[\mathbb{C P}^{1}\right] \in H^{2}(S ; \mathbb{Z})$. Suppose that $S$ is of finite type of order $n \geq 1$ with respect to $w$ and $w+\Sigma$ for the metrics defined by $\Sigma$. Then

$$
0=D_{S}^{(w, \Sigma)}\left(\left(x^{2}-4\right)^{n} z_{\alpha} e^{t \mathbb{C P}^{1}}\right)=<\phi^{w}\left(A,\left(x^{2}-4\right)^{n} e^{t \Delta}\right), e_{\alpha}>
$$

for all $\alpha$. From lemma $21, \phi^{w}\left(A,\left(x^{2}-4\right)^{n} e^{t \Delta}\right)=0$. Then consider $(X, \Sigma)$ permissible, $w \in H^{2}(X ; \mathbb{Z})$ with $w \cdot \Sigma \equiv 1 \quad(\bmod 2), X=X^{o} \cup_{Y} A$. For any $D \in H_{2}(X)$ with $D \cdot \Sigma=1$, we can write $D=D^{o}+\Delta$, so

$$
D_{X}^{(w, \Sigma)}\left(\left(x^{2}-4\right)^{n} e^{t D}\right)=<\phi^{w}\left(X^{o}, e^{t D^{o}}\right), \phi^{w}\left(A,\left(x^{2}-4\right)^{n} e^{t \Delta}\right)>=0 .
$$

We conclude that $D_{X}^{(w, \Sigma)}\left(\left(x^{2}-4\right)^{n} e^{t D}\right)=0$ for all $D \in H_{2}(X)$, i.e. $X$ is of finite type of order at most $n$ with respect to $w$. 
5. Conjecture. Following our results in [18] for the case of genus $g=2$, we propose the following conjecture.

For any $(X, \Sigma)$ permissible, define $\tilde{X}=X \#_{\Sigma} B_{g}$ (we need to fix one identification arbitrarily). Then $b^{+}(\tilde{X})>1$ and also $b_{1}(\tilde{X})=0$ whenever $b_{1}(X)=0$. For any cycle $D^{o} \subset X^{o}$ with $\partial D^{o}=\gamma \subset Y$ an embedded curve (when $X^{o}$ is given a cylindrical end, $\left.D^{o} \cap(Y \times[0, \infty))=\gamma \times[0, \infty)\right)$, we choose cappings $D=D^{o}+D_{B_{g}}^{o}, \partial D_{B_{g}}^{o}=-\gamma$. The cappings have to satisfy the following condition. In $B_{g}=S_{g} \# g \overline{\mathbb{C P}}^{2}$ (see definition 25), we fix an embedded surface $S$ representing $\sigma_{g}$, intersecting $\Sigma_{g}$ transversely in $g$ points. Put $S^{o}=S \cap B^{o}$, then we impose that $D_{B_{g}}^{o} \cdot S^{o}=0$ (the pairing makes sense as long as $\gamma$ and $\partial S^{\circ}$ are disjoint)

ConjeCture 28. Let $\left(X_{1}, \Sigma_{1}\right)$ and $\left(X_{2}, \Sigma_{2}\right)$ be permissible with $X_{i}$ having $b_{1}=0$ (we do not suppose that they are of simple type). Consider $\tilde{X}_{i}$. Then $\tilde{X}_{i}$ are of simple type. Put $\mathbb{D}_{\tilde{X}_{1}}^{w}\left(e^{\alpha}\right)=e^{Q(\alpha) / 2} \sum \tilde{a}_{j, w} e^{\tilde{K}_{j} \cdot \alpha}$ and $\mathbb{D}_{\tilde{X}_{2}}^{w}\left(e^{\alpha}\right)=e^{Q(\alpha) / 2} \sum \tilde{b}_{k, w} e^{\tilde{L}_{k} \cdot \alpha}$. Choose any identification $\phi$ and let $X=X(\phi)=X_{1} \#_{\Sigma} X_{2}$ be the connected sum along $\Sigma$, with the induded homology orientation. Then $X$ is of simple type. Choose $w \in H^{2}(X ; \mathbb{Z}), w_{i} \in H^{2}\left(\tilde{X}_{i} ; \mathbb{Z}\right), i=1,2$ in a compatible way such that $w \cdot \Sigma \equiv 1$ $(\bmod 2), w_{i} \cdot \Sigma \equiv 1 \quad(\bmod 2)$ and $\left.w_{i}\right|_{B_{g}^{\circ}}=k$ P.D. $\left.\left[T_{1}\right]\right|_{B_{g}^{o}}$. For every $D \in H_{2}(X)$, we write $D=D_{1}^{o}+D_{2}^{o}, D_{i}^{o} \subset X_{i}^{o}$, with $\partial D_{1}^{o}=-\partial D_{2}^{o}=\gamma$ disjoint with $\partial S^{o}$ and consider cappings $D_{i} \in H_{2}\left(\tilde{X}_{i}\right)$ of $D_{i}^{o}$ as above, in such a way that the map $D \mapsto\left(D_{1}, D_{2}\right)$ is linear. Then

$$
\begin{aligned}
\mathbb{D}_{X}^{w}\left(e^{t D}\right)= & \epsilon e^{Q(t D) / 2}\left(\sum_{\tilde{K}_{j} \cdot \Sigma=\tilde{L}_{k} \cdot \Sigma=2 g-2}-2^{-3 g+5} \tilde{a}_{j, w} \tilde{b}_{k, w} e^{\left(\tilde{K}_{j} \cdot D_{1}+\bar{L}_{k} \cdot D_{2}\right) t}+\right. \\
& \left.+\sum_{\tilde{K}_{j} \cdot \Sigma=\tilde{L}_{k} \cdot \Sigma=-(2 g-2)}(-1)^{g} 2^{-3 g+5} \tilde{a}_{j, w} \tilde{b}_{k, w} e^{\left(\tilde{K}_{j} \cdot D_{1}+\tilde{L}_{k} \cdot D_{2}\right) t}\right),
\end{aligned}
$$

for $\epsilon=(-1)^{(g-1)\left(w^{2}-w_{1}^{2}-w_{2}^{2}\right) / 2}$.

Acknowledgements: Thanks to my D. Phil. supervisor Simon Donaldson, for many helpful ideas. Also I am very grateful to the Mathematics Department in Universidad de Málaga for their hospitatility and support.

\section{REFERENCES}

[1] P. BRAAM AND S. K. Donaldson, Fukaya-Floer homology and gluing formulae for polynomial invariants, The Floer memorial volume. Progress in mathematics, 133 1994, 257-281.

[2] S. K. Donaldson, On the work of Andreas Floer, Jahresber. Deutsch. Math. Verein, 95 1993, 103 120 .

[3] S. K. Donaldson, Floer homology and algebraic geometry, Vector bundles in algebraic geometry, London Math. Soc. Lecture Notes Series, 208 Cambridge University Press, Cambridge, 1995, 119-138.

[4] S. K. Donaldson And P. B. Kronheimer, The geometry of 4-manifolds, Oxford University Press, 1990.

[5] S. Dostoglou and D. Salamon, Self-dual instantons and holomorphic curves, Annals of Mathematics, 139 1994, 581-640.

[6] R. Fintushel and R. J. Stern, Donaldson invariants of 4-manifolds with simple type, Jour. Diff. Geometry, 42 1995, 577-633.

[7] A. Floer, Symplectic fixed points and holomorphic spheres, Comm. Math. Physics, 120 1989, 575-611.

[8] R. Friedman ANd J. W. Morgan, Smooth four-manifolds and complex surfaces, Springer Verlag, 1994.

[9] A. D. King and P. E. Newstead, On the cohomology ring of the moduli space of rank 2 vector bundles on a curve, Liverpool Preprint, 1994. 
[10] D. Kotschick, $S O(3)$ invariants for 4-manifolds with $b_{2}^{+}=1$, Proc. Lond. Math. Soc. 63 1991, 426-448.

[11] D. Kotschick and J. W. Morgan, $S O(3)$ invariants for 4-manifolds with $b_{2}^{+}=1 \mathrm{II}$, Jour. Differential Geometry, 39 1994, 433-456.

[12] P. B. Kronheimer And T. S. Mrowka, Embedded surfaces and the structure of Donaldson's polynomial invariants, Jour. Differential Geometry, 41 1995, 573-734.

[13] J. W. Morgan AND T. S. MrowkA, The smooth classification of elliptic surfaces, Conference Proceedings and Lecture Notes in Geometry and Topology, IV Geometry, Topology, and Physics for Rauol Bott, International Press, 1995.

[14] J. W. Morgan and Z. Szabó, Embedded genus 2 surfaces in four-manifolds, Duke Math. Jour., 89 1997, 577-602.

[15] J. W. Morgan and Z. Szabó, Embedded tori in four-manifolds, Preprint.

[16] J. W. Morgan, Z. Szabó and C. H. TAubes, A product formula for the Seiberg-Witten invariants and the generalized Thom conjecture, Jour. Diff. Geom., 44 (1996), 706-788.

[17] V. MuÑoz, Oxford D. Phil. Thesis, Gauge Theory and Complex Manifolds, 1996.

[18] V. MuÑoz, Donaldson invariants for connected sums along Riemann surfaces of genus 2, submitted to Topology and Its Applications.

[19] A. Stipsicz, Donaldson series and (-1)-tori, Jour. Reine ange Math. 465 1995, 133-144.

[20] Z. SzABó, Irreducible four-manifolds with small Euler characteristics, Topology. 35 1996, 411426.

[21] M. Thaddeus, Conformal field theory and the cohomology of the moduli space of stable bundles, Jour. Differential Geometry, 35 1992, 131-150.

[22] E. Witten, Monopoles and four-manifolds, Mathematical Research Letters, 1 1994, 769-796. 